\title{
Pyrrolizidine alkaloids in honey: determination with liquid chromatography-mass spectrometry method
}

\author{
Ewelina Kowalczyk, Krzysztof Kwiatek \\ Department of Hygiene of Animal Feedingstuffs \\ National Veterinary Research Institute, 24-100 Pulawy, Poland \\ ewelina.kowalczyk@piwet.pulawy.pl
}

Received: February 8, 2018

Accepted: June 20, 2018

\begin{abstract}
Introduction: Pyrrolizidine alkaloids (PAs) are probably the most widespread toxins of natural origin. More than 6,000 plant species produce these toxic compounds. Bees can forage on flowers of plants producing PAs, which leads to contamination of honey with the toxic compounds. To determine the contamination of honey with PAs, a sensitive method based on liquid chromatography coupled with mass spectrometry has been developed. Material and Methods: PAs were extracted with $0.05 \mathrm{M}$ sulphuric acid and purified with MCX cartridges. A solvent mixture consisting of ethyl acetate, methanol, acetonitrile, ammonia, and triethylamine $(8: 1: 1: 0.1: 0.1, \mathrm{v} / \mathrm{v})$ was used to wash alkaloids from the cartridges. After evaporation the residues were reconstituted in water and methanol mixture and subjected to LC-MS analysis. Results: The developed method was validated according to SANTE/11945/2015 requirements. The recovery was from $80.6 \%$ to $114.5 \%$. The repeatability ranged from $2.3 \%$ to $14.6 \%$, and the reproducibility was from $4.9 \%$ to $17.7 \%$. Conclusions: A new method for the determination of PAs in honey has been developed and validated. All evaluated parameters were in accordance with the SANTE/11945/2015 guidance document. Out of 50 analysed honey samples, $16(32 \%)$ were positive for the content of at least one PA.
\end{abstract}

Keywords: honey, food, pyrrolizidine alkaloids, LC-MS.

\section{Introduction}

Pyrrolizidine alkaloids (PAs) are one of the most common groups of natural toxins. They are produced by a wide variety of plants as a chemical defence against herbivores (4). Plants containing PAs often grow undesired in agricultural production systems, posing a risk of contamination of feeds and crops (9). Bees often forage on the flowers of plants producing PAs, which leads to contamination of honey with the toxic compounds (6).

The toxicity of PAs in humans is documented in a series of case reports of intoxication following ingestion of PAs containing herbal medicines and teas, and outbreak cases including deaths caused by the consumption of grain contaminated with PAs containing weeds (15). However, not all PAs exert toxicity. Only 1,2-unsaturated alkaloids are pro-toxins which can be converted into toxic metabolites in the liver. The conversion is triggered by the cytochrome $\mathrm{P} 450$ monooxygenases located primarily in hepatocytes. As a result, reactive electrophilic pyrrolic metabolites capable of binding to proteins and nucleic acids are created $(9,22)$.

PAs can be a cause of acute and chronic intoxication. Acute poisoning with PAs in humans is more associated with liver damage (13). In the case when the small amounts of dehydro PAs are regularly delivered via diet, cancer, pulmonary arterial hypertension, and cirrhosis are more likely to occur (10).

Humans can ingest PAs unintentionally via consumption of various products. These toxic compounds can be consumed with grains contaminated with PA-producing plants, vegetable harvests with similar-looking weeds (e.g. ragwort), herbal preparations, teas, honey, pollen contaminated with PAs (4) and other food such as milk or eggs $(26,27)$.

Even though the toxicity of PAs has been well documented and high concentrations of PAs have been detected in various products, there is no official limit for the maximum allowable level of PAs in food and feed.

In Poland the production of honey is systematically increasing. The same trend can be observed in its consumption. Honey is consumed in its pure form or as 
an ingredient of breakfast cereals, sweets, or baked products. However, it has been proved that honey can be contaminated with PAs, and in some cases, high concentration of the alkaloids have been detected $(1,3$, $7,8,19,22)$. To increase consumer protection by minimising dietary exposure to these toxins, it was suggested that all honeys need to be assessed for their content of PAs $(8,11,28)$. In a European Food Safety Authority (EFSA) report it was suggested that new and sensitive analytical methods enabling PAs determination should be developed (13).

Hitherto, different analytical methods have been described for the determination of PAs in honey. Most of them were based on the LC-MS/MS analysis and for the purification of extracts mostly cation exchange cartridges were used combined with ammonia in methanol elution of PAs. However, the purification of the extracts is not efficient, especially when LC-MS is used for the instrumental analysis, and many problems concerning the appropriate identification and quantification of PAs can occur. That is why a new sensitive analytical method providing effective clean-up of honey extracts has been developed.

Based on the EFSA recommendations and on the results of the occurrence of PAs in honey of European origin reported by other authors $(8,13,17,25)$, the ten most often detected alkaloids were selected for the study. The compounds designated were Senecioninetype PAs: jacobine, retrorsine, senecionine, and seneciphylline; Lycopsamine-type PAs: lycopsamine, intermedine, and echimidine; and Heliotrine-type PAs: heliotrine and lasiocarpine. Senkirkine was also included, as it was found that together with echimidine, echimidine $N$-oxide, heliotrine, lycopsamine, retrorsine, senecionine, and seneciphylline it constituted around $75 \%-90 \%$ of the total PAs measured in honey (14). The $\mathrm{N}$-oxides are reduced with the zinc dust, hence the final determined concentration reflects the content of both free base and $N$-oxide forms.

\section{Material and Methods}

Chemicals and reagents. Formic acid and zinc dust were from Sigma-Aldrich (USA), ethyl acetate and triethylamine were Merck (Germany) products, and methanol and acetonitrile were from the J.T. Baker catalogue (the Netherlands). Sulphuric acid (95\%) was purchased from Chempur (Poland) and 25\% ammonia solution was sourced from POCH (Poland). The Milli-Q water purification system (Millipore, USA) was used to obtain pure water. Oasis MCX solid phase extraction (SPE), mixed-mode polymeric cartridges were from Waters (USA), Bond Elut Plexa PCX and HF Bond Elut-SCX cartridges came from Agilent (USA), and Strata SCX and polymeric Strata XC cartridges were ordered from Phenomenex (USA). All cartridges were of $500 \mathrm{mg}$ bed weight and $6 \mathrm{ml}$ volume. Analytical standards of intermedine, lycopsamine, jacobine, retrorsine, heliotrine, seneciphylline, senecionine, echimidine, senkirkine, lasiocarpine, $N$-oxides of senecionine, echimidine and retrorsine were purchased from PhytoLab (Germany).

Standard solutions. Stock standard solutions of intermedine, lycopsamine, jacobine, retrorsine, heliotrine, seneciphylline, senecionine, echimidine, senkirkine, lasiocarpine and were prepared at a concentration of $1 \mathrm{mg} \mathrm{mL}^{-1}$ in methanol and stored at $-18^{\circ} \mathrm{C}$. A mixed standard solution of $1 \mu \mathrm{g} \mathrm{mL} \mathrm{m}^{-1}$ was prepared by mixing the appropriate volume of each stock standard solution and subsequently diluting serially. The mixed standard solution was stored between 2 and $4{ }^{\circ} \mathrm{C}$.

Honey samples. The honey samples were from Poland and were collected directly from apiaries in 2017. The honey analysed included polyfloral, acacia, rape, and honeydew. Part of the samples were of unknown type.

Sample preparation. $10 \mathrm{~g}$ of homogenised honey was weighed into a $50 \mathrm{~mL}$ polypropylene tube and dissolved in $20 \mathrm{~mL}$ of $0.05 \mathrm{M}$ sulphuric acid. About $1 \mathrm{~g}$ of zinc dust was added to reduce $N$-oxides, and samples were left overnight. On the next day, the samples were slowly shaken for $0.5 \mathrm{~h}$, subsequently centrifuged $(4,000 \mathrm{~g}$, $10 \mathrm{~min}$ ) and passed through cellulose filters, then purified with the use of solid phase extraction. The samples were applied on MCX cartridges preconditioned with $9 \mathrm{~mL}$ of methanol and equilibrated with $9 \mathrm{~mL}$ of $0.05 \mathrm{M} \mathrm{H}_{2} \mathrm{SO}_{4}$. A total of $12 \mathrm{~mL}$ of $\mathrm{H}_{2} \mathrm{O}$ and $12 \mathrm{~mL}$ of $\mathrm{MeOH}$ were used for washing purposes, subsequently cartridges were vacuum dried for $2 \mathrm{~min}$, and $6 \mathrm{~mL}$ of ethyl acetate were added. The elution of the alkaloids was done with $12 \mathrm{~mL}$ of solvent mixture consisting of ethyl acetate, methanol, acetonitrile, ammonia, and triethylamine $(8: 1: 1: 0.1: 0.1 \mathrm{v} / \mathrm{v})$. After evaporation at $40^{\circ} \mathrm{C}$ in a nitrogen stream the residues were reconstituted in $0.2 \mathrm{~mL}$ of water and $0.2 \mathrm{~mL}$ of methanol and passed through $0.2 \mu \mathrm{m}$ PVDF syringe filters.

LC-MS parameters. HP 1200 Series separation modules from Agilent Technologies (USA) were used for the instrumental analysis. The modules included a degasser system, binary pump, automatic injector, column thermostat, and single quadrupole mass spectrometry detector (Agilent Technologies 6140). The alkaloids were separated on a Gemini $3 \mu \mathrm{m}$ NX-C18, $150 \mathrm{~mm} \times 4.6 \mathrm{~mm}$ column, (Phenomenex, USA) coupled with a C18 guard column (Phenomenex). The column thermostat was set at $30^{\circ} \mathrm{C}$. The separation of the compounds was carried out in a gradient mode: $0-2 \mathrm{~min}$, $5.5 \% \mathrm{~B} ; 2-8 \mathrm{~min}, 12 \% \mathrm{~B} ; 8-11 \mathrm{~min}, 20 \% \mathrm{~B} ; 11-12 \mathrm{~min}$, $30 \% \mathrm{~B} ; 12-15 \mathrm{~min}, 40 \% \mathrm{~B} ; 17-16 \mathrm{~min}, 70 \% \mathrm{~B}$; $16-17 \mathrm{~min}$, $85 \% \mathrm{~B}$; and $17-23 \mathrm{~min}, 5.5 \% \mathrm{~B}$. The mobile phase consisted of $0.2 \%$ formic acid in water $(\mathrm{A})$ and a mixture of methanol and acetonitrile $(1: 1, \mathrm{v} / \mathrm{v})(\mathrm{B})$. The flow rate was $0.6 \mathrm{~mL} \mathrm{~min}^{-1}$ and the injection volume was $5 \mu \mathrm{L}$.

Electrospray ionisation (ESI) was in a positive mode, the capillary voltage was set at $2,000 \mathrm{~V}$, nebulising pressure was 35 psi, drying gas flow was set at $11.0 \mathrm{~L} \mathrm{~min}{ }^{-1}$, and temperature of drying gas was $300^{\circ} \mathrm{C}$. The fragmentor voltage was set at $100 \mathrm{~V}$ for all target alkaloids, and selected ion monitoring was used for the detection. Monitored, protonated molecular $(\mathrm{M}+\mathrm{H})^{+}$ions $(\mathrm{m} / \mathrm{z})$ are listed in Table 1. 
Table 1. Selected $(\mathrm{m} / \mathrm{z})$ ions monitored and retention times of the monitored alkaloids. Coefficients of determination obtained for matrix calibration curves in a concentration range corresponding to $0-50 \mu \mathrm{g} \mathrm{kg}^{-1}$ and matrix effect results

\begin{tabular}{lllll}
\hline PA & $\mathrm{m} / \mathrm{z}$ & $\mathrm{RT}$ & $\mathrm{R}^{2}$ & $\mathrm{ME}(\%)$ \\
\hline Intermedine & 300.1 & 6.16 & 0.997 & 105.5 \\
Lycopsamine & 300.0 & 6.38 & 0.99 & 116.5 \\
Jacobine & 352.1 & 6.91 & 0.998 & 119.2 \\
Retrorsine & 352.1 & 8.31 & 0.998 & 117.4 \\
Heliotrine & 314.1 & 8.63 & 0.998 & 119.9 \\
Seneciphylline & 334.1 & 9.33 & 0.998 & 119.2 \\
Senecionine & 336.1 & 11.14 & 0.994 & 122.6 \\
Echimidine & 398.0 & 13.18 & 0.998 & 117.1 \\
Senkirkine & 366.1 & 13.37 & 0.995 & 106.2 \\
Lasiocarpine & 412.1 & 15.11 & 0.996 & 107.5 \\
\hline
\end{tabular}

PA - pyrrolizidine alkaloid, RT - retention time, $\mathrm{R}^{2}$ - coefficient of determination, $\mathrm{ME}$ - matrix effect

Identification and quantification. Identification was made on the basis of retention time and the protonated molecular ion $(\mathrm{M}+\mathrm{H})^{+}$of monitored compounds.

Quantification was achieved using matrix calibration curves prepared by adding appropriate amounts of mixed reference standard solutions to blank honey before the extraction procedure. Calibration curves at the concentration range $0-50 \mu \mathrm{g} \mathrm{kg}^{-1}$ were constructed by plotting the pyrrolizidine alkaloid (PA) peak area versus its concentrations.

Method validation. The validation of the method was performed according to the SANTE/11945/2015 guidance document (29). Parameters such as method linearity, recovery, repeatability, reproducibility, specificity, limit of quantification (LOQ), robustness, stability of PAs, and matrix effect were evaluated during the validation process. Buckwheat honey was used as the blank matrix, as no alkaloids were determined in this type of honey.

For evaluation of the linearity of the method, matrix calibration curves were used. Blank honey samples before the extraction treatment were fortified with the mix standard solution to obtain appropriate concentrations corresponding to $0,1,2,5,10,20$, and $50 \mu \mathrm{g} \mathrm{kg}^{-1}$, and were analysed in triplicate.

To evaluate the method's recovery and precision, honey blanks were spiked at different levels corresponding to the concentrations 1,10 , and $50 \mu \mathrm{g} \mathrm{kg}^{-1}$, with a set of six samples for each level. The samples were analysed with the same instrument and by the same operator. The relative standard deviation of the results (RSD) (\%) was assigned as the expression of the repeatability. For the determination of reproducibility, other sets of samples were spiked at the same concentrations as for repeatability and analysed on different days with the same instrument. The reproducibility was also calculated as relative standard deviation $(\%)$.

For the selectivity determination, an analysis of a set of blank honey samples was performed in order to check the presence of interfering compounds.

The limit of quantification was established on the basis of the SANTE guidance document (29), according to which the LOQ was assumed to be the lowest spike level meeting the method performance criteria for trueness and precision.
Matrix effect was evaluated by dividing a slope of matrix-matched calibration by a slope of standard calibration in solvent and expressing the result as a percentage.

A stability test was performed on purified extracts of honey blanks that were contaminated at $20 \mu \mathrm{g} \mathrm{kg}^{-1}$ before the extraction procedure. The dry extracts were stored at $-18^{\circ} \mathrm{C}, 4^{\circ} \mathrm{C}$, and $20^{\circ} \mathrm{C}$. The extracts were subsequently analysed on days $1,2,4,8,14$, and 30 .

To determine the robustness of the method, the Youden procedure was applied (30). Blank honey samples fortified at the concentration of $20 \mu \mathrm{g} \mathrm{kg}^{-1}$ were analysed in order to evaluate the effect of the seven chosen variables which were altered. The effect of percentage of sulphuric acid (0.05 $\mathrm{M}$ and $0.1 \mathrm{M})$, volume of elution mixture $(12 \mathrm{~mL}$ and $10 \mathrm{~mL})$, temperature of evaporation $\left(40^{\circ} \mathrm{C}\right.$ and $\left.45^{\circ} \mathrm{C}\right)$, percentage of methanol in the mobile phase $(50 \%$ and $47 \%)$, percentage of acetonitrile in the mobile phase (50\% and $47 \%)$, thermostat temperature $\left(30^{\circ} \mathrm{C}\right.$ and $\left.33^{\circ} \mathrm{C}\right)$, and flow rate $\left(0.6 \mathrm{~mL} \mathrm{m^{-1 }}\right.$ and $0.57 \mathrm{~mL} \mathrm{~min}^{-1}$ ) were evaluated. Student's $t$-test was used to determine the impact of changes in individual parameters on the results.

\section{Results}

Validation results. Good linearity over the concentration range $0-50 \mu \mathrm{g} \mathrm{kg}^{-1}$ was observed for the target PAs. The determination coefficients $\mathrm{R}^{2}$ calculated for each of the matrix calibration curves were equal or greater than 0.99 (Table 1). Matrix effect was noticeable in the case of most of the analysed alkaloids, especially for jacobine, heliotrine, seneciphylline, echimidine, and senecionine (Table 1).

Validation parameters (Table 2) were determined on the basis of analysis of sets of blank honey samples spiked at the levels corresponding to concentrations 1 , 10 , and $50 \mu \mathrm{g} \mathrm{kg}^{-1}$. Obtained recovery values were from $80.6 \%$ to $114.5 \%$. The repeatability was calculated as relative standard deviation and ranged from $2.3 \%$ to $14.6 \%$, and the reproducibility was in the range of $4.9 \%$ to $17.7 \%$. The method can be perceived to be selective, as no interfering peaks were determined in the retention time of the selected alkaloids (Fig. 1). 
Table 2. Validation parameters evaluated for ten alkaloids in honey matrix

\begin{tabular}{|c|c|c|c|c|c|c|c|c|c|}
\hline \multirow[b]{3}{*}{ PA } & \multicolumn{3}{|c|}{$\begin{array}{l}\text { Concentration level } \\
\left(\mu \mathrm{g} \mathrm{kg}^{-1}\right)\end{array}$} & \multicolumn{3}{|c|}{$\begin{array}{c}\text { Concentration level } \\
\left(\mu \mathrm{g} \mathrm{kg}^{-1}\right)\end{array}$} & \multicolumn{3}{|c|}{$\begin{array}{c}\text { Concentration level } \\
\left(\mu \mathrm{g} \mathrm{kg}^{-1}\right)\end{array}$} \\
\hline & 1 & 10 & 50 & 1 & 10 & 50 & 1 & 10 & 50 \\
\hline & \multicolumn{3}{|c|}{$\begin{array}{c}\text { Recovery } \\
(\%)\end{array}$} & \multicolumn{3}{|c|}{$\begin{array}{l}\text { Repeatability } \\
\text { RSD (\%) }\end{array}$} & \multicolumn{3}{|c|}{$\begin{array}{l}\text { Reproducibility } \\
\text { RSD (\%) }\end{array}$} \\
\hline Intermedine & 80.6 & 84.6 & 96.3 & 14.6 & 6.0 & 5.4 & 17.7 & 16.6 & 10.1 \\
\hline Lycopsamine & 108.2 & 97.4 & 100.6 & 11.1 & 9.1 & 2.5 & 16.3 & 9.2 & 7.1 \\
\hline Jacobine & 90.7 & 99.3 & 97.5 & 10.9 & 9.7 & 4.5 & 15.2 & 11.5 & 4.9 \\
\hline Retrorsine & 98.2 & 105.5 & 98.6 & 13.6 & 5.2 & 7.5 & 16.8 & 7.3 & 8.5 \\
\hline Heliotrine & 80.9 & 101.4 & 95.6 & 8.5 & 4.0 & 4.3 & 15.0 & 7.3 & 5.1 \\
\hline Seneciphylline & 108.1 & 101.5 & 111.6 & 7.3 & 5.3 & 2.5 & 12.8 & 11.2 & 15.6 \\
\hline Senecionine & 87.2 & 106.9 & 102.4 & 7.5 & 7.2 & 2.3 & 10.3 & 11.2 & 7.6 \\
\hline Echimidine & 103.9 & 109.4 & 114.5 & 8.1 & 5.6 & 6.6 & 15.2 & 17.0 & 13.0 \\
\hline Senkirkine & 88.0 & 104.5 & 100.5 & 9.1 & 2.9 & 4.4 & 11.8 & 9.5 & 5.3 \\
\hline Lasiocarpine & 82.2 & 108.9 & 105.6 & 2.9 & 12.4 & 3.1 & 12.1 & 12.5 & 7.9 \\
\hline
\end{tabular}

Table 3. Summary of detected PAs

\begin{tabular}{lcccc}
\hline PA & $\begin{array}{c}\text { Number } \\
\text { of samples }\end{array}$ & $\begin{array}{c}\text { Mean concentration } \\
\left(\mu \mathrm{g} \mathrm{kg}^{-1}\right)\end{array}$ & $\begin{array}{c}\text { Median } \\
\left(\mu \mathrm{g} \mathrm{kg}^{-1}\right)\end{array}$ & $\begin{array}{c}\text { Min.-Max content } \\
(\mu \mathrm{g} \mathrm{kg})\end{array}$ \\
\hline Intermedine & 4 & 4.8 & 1.9 & $<\mathrm{LOQ}-14.7$ \\
Lycopsamine & 5 & 9.4 & 3.0 & $1.7-32.9$ \\
Retrorsine & 4 & 1.1 & 1.2 & $<$ LOQ-1.8 \\
Senecionine & 3 & 1.0 & $<$ LOQ & $<$ LOQ-2.9 \\
Echimidine & 7 & 3.3 & 3.8 & $1.4--5.2$ \\
\hline
\end{tabular}
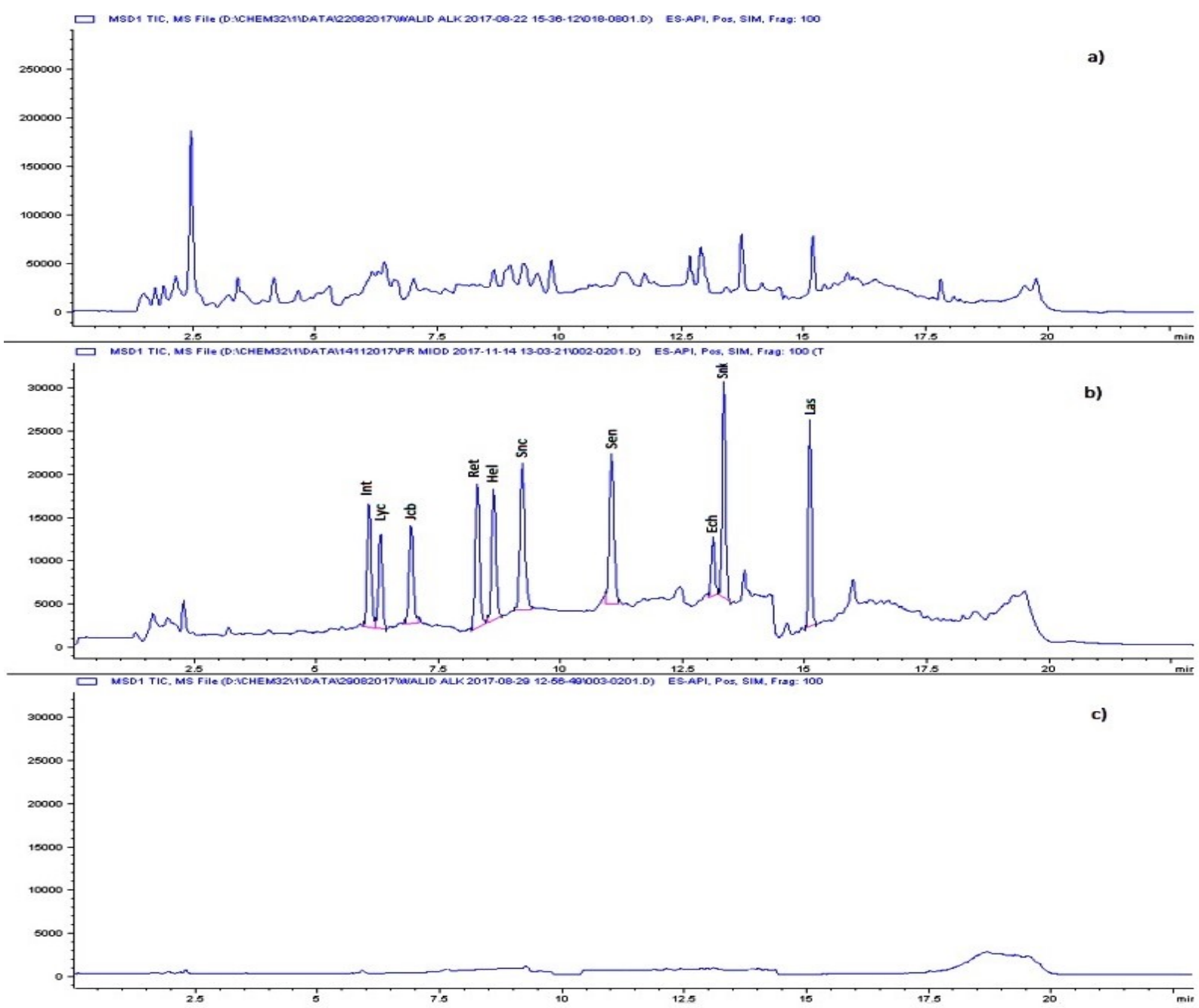

Fig. 1. SIM chromatograms of honey samples obtained for a) HF Bond cartridge and $3 \%$ ammonia in methanol elution (PAs concentration $1 \mu \mathrm{g} \mathrm{kg}^{-1}$ ); b) MCX cartridge and new elution mixture consisting of ethyl acetate, methanol, acetonitrile, ammonia, and triethylamine (8:1:1:0.1:0.1, v/v) elution (PAs concentration $1 \mu \mathrm{g} \mathrm{kg}^{-1}$ ); c) blank sample

Int - Intermedine, Lyc - lycopsamine, Jcb - jacobine, Ret - retrorsine, Hel - heliotrine, Snc - seneciphylline, Sen - senecionine, Ech - echimidine, Skn - senkirkine, Las - lasiocarpine 


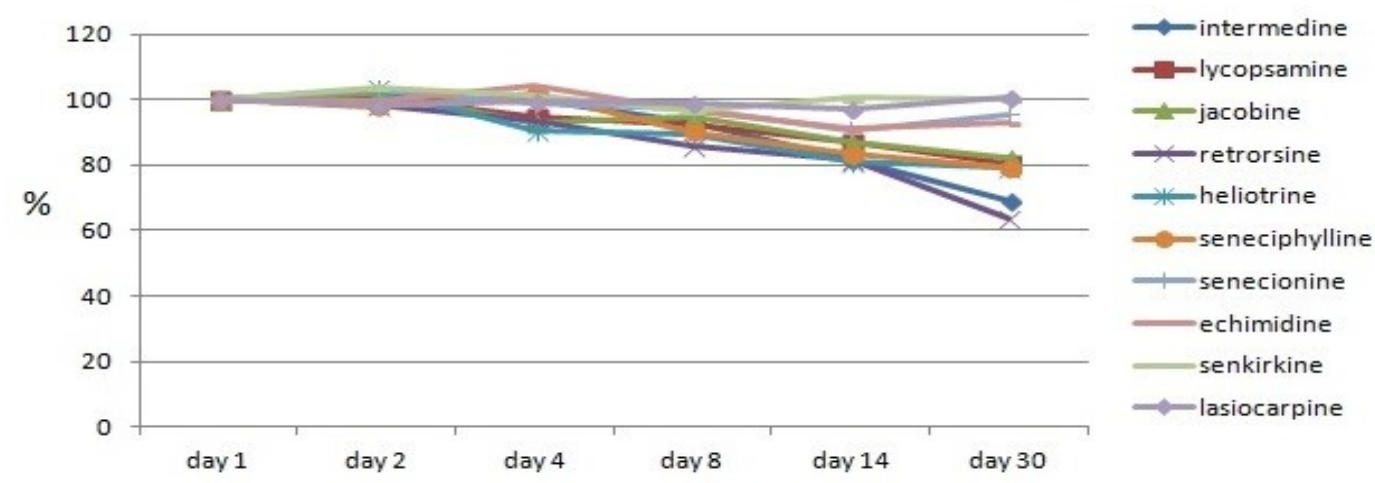

Fig. 2. Stability results of the target PAs in dry SPE-purified extracts of honey matrix, stored at $-18^{\circ} \mathrm{C}$ for 30 days

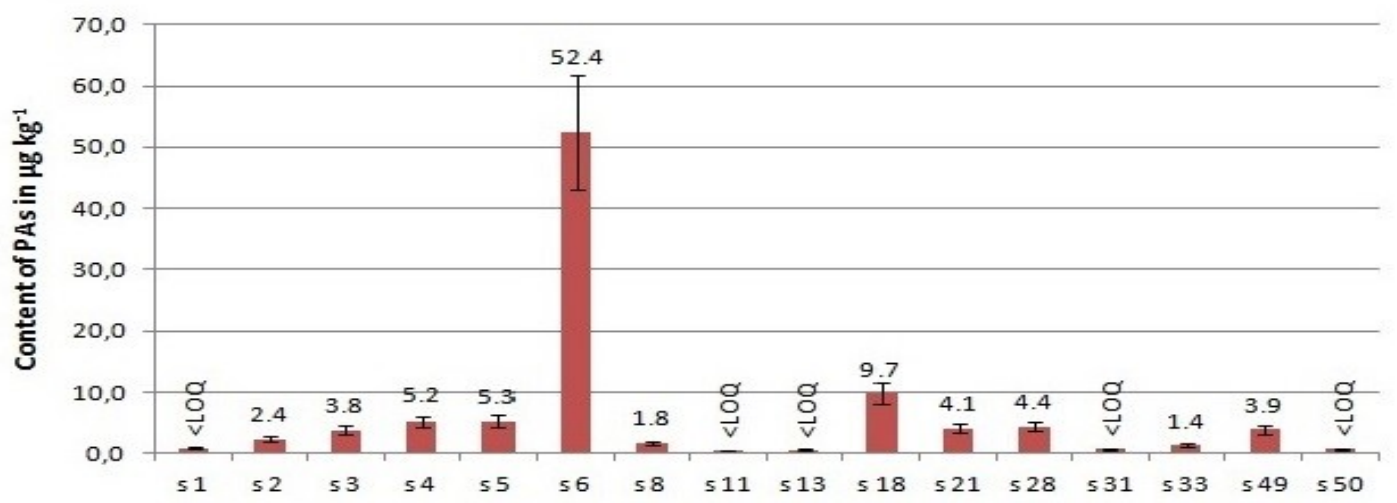

Fig. 3. The content of pyrrolizidine alkaloids in positive honey samples

The robustness test revealed that chosen variables do not affect the analysis. The calculated standard deviation was not significantly higher than the standard deviation of the method carried out under withinlaboratory reproducibility conditions. To determine the impact of changes in individual parameters on the result of the analysis, Student's $t$-test was used and no significant statistical differences were determined. Changes in composition of the mobile phase did not affect the retention times; however, slight shifts could be observed with higher thermostat temperature and lower flow rate.

The stability test revealed that dry extracts of honey can be stored at $-18^{\circ} \mathrm{C}$ and $4^{\circ} \mathrm{C}$ for a week without a significant change in PAs concentrations (Fig. 2, data shown only for $-18^{\circ} \mathrm{C}$ ).

The limit of quantification of the developed method for the determination of PAs in honey was established at $1 \mu \mathrm{g} \mathrm{kg}^{-1}$ for all individual alkaloids, as it was the lowest spiked level with the recovery and precision values that were in the range fixed in the SANTE guidance document (Table 2).

Real samples application. The developed method has been applied to the analysis of 50 honey samples. Within the samples, $16(32 \%)$ were contaminated with at least one of the alkaloids, however, in five samples the determined concentrations were below LOQ (Fig. 3). In five samples more than one alkaloid was found. Among detected PAs, echimidine was the most abundant alkaloid, being present in $43.8 \%$ of the positive samples in the concentration range of $1.4-5.2 \mu \mathrm{g} \mathrm{kg}^{-1}$ (Table 3). Lycopsamine and intermedine were detected in $31.3 \%$ and $25 \%$ of the positive honey samples, respectively. Retrorsine was detected in $25 \%$ and senecionine was present in $18.8 \%$ of all positive samples.

The total content of alkaloids in particular samples ranged from 1.4 to $52.4 \mu \mathrm{g} \mathrm{kg}^{-1}$ (Fig. 3). The mean and median values (calculated for PA positive samples and without values <LOQ) were 8.6 and $4.1 \mu \mathrm{g} \mathrm{kg} \mathrm{kg}^{-1}$, respectively. When all samples were included into the calculations, the mean concentration was $2.0 \mu \mathrm{g} \mathrm{kg}^{-1}$.

\section{Discussion}

Method development and validation. Honey is considered a difficult and complex matrix of variable composition. The ratio of different substances such as proteins, minerals, organic acids, etc. can be influenced by the plant source and other factors such as seasonal and environmental conditions (12). Additionally, the fact that PAs can occur in very low concentrations poses a challenge as far the analysis of the compounds is concerned.

During the development of this LC-MS method various parameters were optimised to deliver the most 
effective performance in recovery, precision, and chromatographic separation. Even though the $\mathrm{N}$-oxides can be directly determined using liquid chromatography, it was decided to reduce them to their free base form. As in the case of a previous gas chromatography-mass spectrometry method (21), the influence of the reduction reaction was also revealed in the purer chromatographic image. Reduction time was optimised with the use of three $N$-oxides: retrorsine, senecionine, and echimidine. The reduction efficiency was checked for 1.5, 2, 3, 4, 5, and $24 \mathrm{~h}$. Almost $100 \%$ conversion of $\mathrm{N}$-oxides of senecionine and echimidine and over $80 \%$ conversion of retrorsine $\mathrm{N}$-oxide was achieved after the $24 \mathrm{~h}$ reduction period.

For the clean-up of basic amine compounds from complex matrices, cation exchange sorbents have been widely used. Cation exchange SPE cartridges were the choice of most of the authors describing determination of PAs in honey. They also used ammonia solution of different concentrations in methanol for the elution of PAs from the cartridges and LC-MS/MS for the instrumental analysis $(2,3,5,8,18,20,22,23)$. However, the eluate was not always properly purified, which could lead to problems such as ion suppression or enhancement or erroneous identification of the target compounds. Moreover, in the case of LC-MS analysis, the identification of the alkaloids at low concentrations was impossible when ammonia in methanol was used for PAs elution.
In the previously conducted study, a new elution mixture consisting of ethyl acetate, methanol, ammonia and triethylamine $(8: 2: 0.1: 0.1, \mathrm{v} / \mathrm{v})$ was developed for the PAs elution from cation exchange cartridges (21). As the technique has been changed, the SPE clean-up procedure had to be re-optimised. The optimisation was performed to investigate the impact of the previously developed and modified elution mixture (Table 4) on the purification of honey extracts along with the recovery of 10 alkaloids using different cation exchange SPE sorbents: MCX, PCX, Strata XC, SCX, and HF Bond.

In general, comparable recoveries were obtained for most of the solvents mixtures. However, the purity of the chromatograms was also a very important factor influencing the choice of the final elution mixture and SPE cartridge. An increased volume of $\mathrm{MeOH}$ or $\mathrm{NH}_{4} \mathrm{OH}$ caused increased elution of impurities, which affected the quality of the chromatograms, whereas the introduction of $1 \mathrm{~mL}$ of acetonitrile into the elution mixture and a decrease in the volume of methanol allowed very pure chromatograms to be obtained. As far as the cartridges are concerned, good recovery rates were obtained with MCX, PCX, and SCX cartridges (Fig. 4). In the case of HF cartridge, intermedine and lycopsamine were recovered to much lower extents. Cartridges such as SCX, Strata XC, and PCX caused additional problems related to clogging and prolonging the time of SPE procedure. A much worse purification effect was obtained with PCX and Strata XC comparing to other cartridges.

Table 4. Combinations of solvents used during optimisation of solid phase extraction step. Recovery rates obtained for target alkaloids at the concentration of $20 \mu \mathrm{g} \mathrm{kg}^{-1}$

\begin{tabular}{lll}
\hline Solvents & Ratio $(\mathrm{v} / \mathrm{v})$ & Recovery $(\%)$ \\
\hline EtAc $: \mathrm{MeOH}: \mathrm{NH}_{4} \mathrm{OH}$ & $8: 2: 0.2$ & $77-93$ \\
EtAc $: \mathrm{MeOH}: \mathrm{NH}_{4} \mathrm{OH}: \mathrm{TEA}$ & $8: 2: 0.1: 0.1$ & $84-98$ \\
EtAc $: \mathrm{MeOH}: \mathrm{ACN}: \mathrm{NH}_{4} \mathrm{OH}:$ TEA & $8: 1: 1: 0.1: 0.1$ & $79-105$ \\
EtAc $: \mathrm{MeOH}: \mathrm{ACN}: \mathrm{NH}_{4} \mathrm{OH}:$ TEA & $7: 1.5: 1.5: 0.1: 0.1$ & $78-92$ \\
EtAc $: \mathrm{MeOH}: \mathrm{ACN}: \mathrm{NH}_{4} \mathrm{OH}:$ TEA & $8: 1: 1: 0.2: 0.1$ & $76-97$ \\
EtAc $: \mathrm{MeOH}: \mathrm{ACN}: \mathrm{NH}_{4} \mathrm{OH}:$ TEA & $3: 3: 3: 0.1: 0.1$ & $61-93$ \\
$\mathrm{MeOH}: \mathrm{NH}_{4} \mathrm{OH}$ & $11: 1.5$ & $48-110$ \\
\hline
\end{tabular}

EtAc - ethyl acetate, $\mathrm{MeOH}$ - methanol, $\mathrm{ACN}$ - acetonitrile, $\mathrm{NH}_{4} \mathrm{OH}$ - ammonia solution, TEA - triethylamine

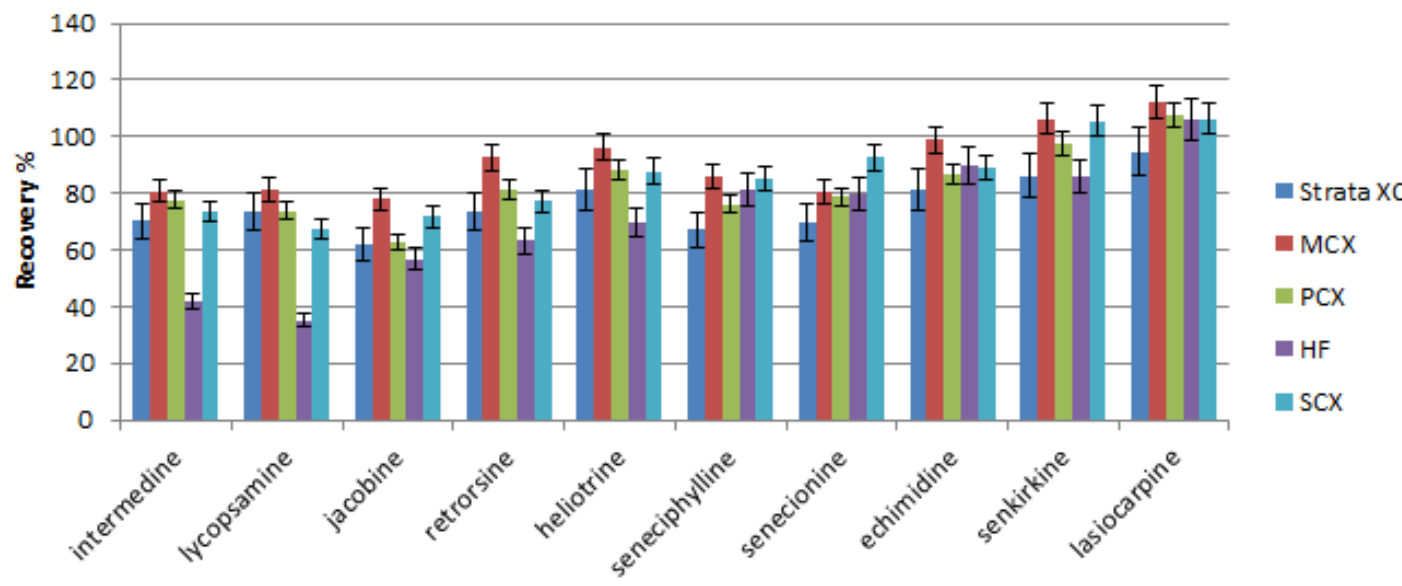

Fig. 4. Recoveries of the alkaloids from different SPE cartridges eluted with the new solvents mixture consisting of ethyl acetate, methanol, acetonitrile, ammonia, and triethylamine $(8: 1: 1: 0.1: 0.1, \mathrm{v} / \mathrm{v})$ 
The best results concerning recoveries of target compounds and purity of chromatograms were obtained with the elution mixture consisting of ethyl acetate, methanol, acetonitrile, ammonia, and triethylamine (8:1:1:0.1:0.1 v/v) in combination with MCX cartridges.

For comparison purposes and to highlight the efficiency of clean-up with the new elution mixture and MCX cartridge, the results of purification obtained with HF Bond cartridge and 3\% ammonia solution in methanol have been presented in Fig. 1. The HF cartridge was chosen for the comparison as it was often used by other authors $(3,7,8,20)$.

To achieve the best chromatographic performance different columns and mobile phases of variable composition were evaluated. The chromatographic columns assessed were Zorbax XDB C18, $150 \mathrm{~mm} \times 4.6 \mathrm{~mm}$, $5 \mu \mathrm{m}$ (Agilent); Gemini NX-C18 $150 \mathrm{~mm} \times 4.6 \mathrm{~mm}$, $3 \mu \mathrm{m}$ (Phenomenex); Kinetex C8, $100 \mathrm{~mm} \times 4.6 \mathrm{~mm}$, $2.6 \mu \mathrm{m}$ (Phenomenex); and Zorbax ODS C18, $250 \mathrm{~mm}$ $\times 4.6 \mathrm{~mm}, 5 \mu \mathrm{m}$ (Agilent). Tested combinations of mobile phases included: $0.1 \%-0.5 \%$ formic acid in water (A) with $\mathrm{ACN}(\mathrm{B}) ; 0.1 \%-0.5 \%$ formic acid in water (A) with $\mathrm{MeOH}(\mathrm{B}) ; 0.1 \%-0.5 \%$ formic acid in water (A) with ACN:MeOH (1:1, v/v) (B); 0.1\%-0.3\% formic acid in water (A) with $0.1 \%-0.3 \%$ formic acid in $\mathrm{ACN}(\mathrm{B})$; and $0.1 \%-0.3 \%$ formic acid in water (A) with $0.1 \%-0.3 \%$ formic acid in $\mathrm{MeOH}$ (B). However, none of the combinations of mobile phases containing only methanol or only acetonitrile as the organic phase allowed good separation of the analysed compounds. The separation of 10 alkaloids including two enantiomeric structures of intermedine and lycopsamine that usually co-elute, was achieved with a Gemini NX-C18 column and mobile phase consisting of $0.2 \%$ formic acid in water (A) and a mixture methanol:acetonitrile (1:1, v/v) (B), (Fig. 1). Parameters affecting the mass spectrometry detection were optimised with the use of flow injection analysis.

The method was successfully validated according to the stipulations of the SANTE/11945/2015 guidance document (29). The method proved to be linear in the concentration range $0-50 \mu \mathrm{g} \mathrm{kg}^{-1}$ and selective as no interfering peaks were determined in the retention times of monitored alkaloids. The recovery was in the range of $80.6 \%-114.5 \%$, which was in compliance with SANTE requirements, according to which the recovery should be in the range of $70 \%-120 \%$. The obtained results were also comparable with recoveries obtained by other authors. Lucatello et al. (22) reported recoveries ranging from $82.7 \%$ to $104.2 \%$ and Griffin et al. (18) obtained recoveries in a range of $82 \%-112 \%$. Recoveries reported by Martinello et al. (24) ranged from $92 \%$ to $115 \%$. Some authors reported slightly lower rates of recoveries ranging from $70 \%$ to $90 \%(1,3)$. Repeatability and reproducibility values were below $20 \%$ and were in the ranges of $2.3 \%-14.6 \%$ and $4.9 \%-17.7 \%$, respectively. Other authors reported similar values. Bodi et al. (3) obtained values $2 \%-13 \%$ and $2 \%-11 \%$, Lucatello et al. (22) obtained $0.8 \%-7.9 \%$ and $3.1 \%-14.2 \%$, Griffin et al. (18) reported $1.1 \%-6.3 \%$ and $2.0 \%-14.9 \%$, and Martinallo et al. (24) reported ranges of $0.9 \%-15.1 \%$ and $1.1 \%-15.6 \%$ for repeatability and reproducibility, respectively.

The developed method proved to be robust to small changes in performance parameters. The matrix effect was noticeable for most of the analysed compounds as it was almost $120 \%$, and in the case of senecionine exceeded this value.

According to an EFSA report of 11 July, 2012 (16), the relevant LOQ to be achieved for the individual PAs in honey was $1 \mu \mathrm{g} \mathrm{kg}^{-1}$. The limit of quantification of the developed method for the determination of PAs in honey was established at $1 \mu \mathrm{g} \mathrm{kg}^{-1}$ for all individual alkaloids, which fulfils the requirements. Obtained LOQ values for the developed LC-MS method are also very comparable or even lower than these reported by other authors for LC-MS/MS methods which ranged from 1 to $68.0 \mu \mathrm{g} \mathrm{kg}^{-1}$ for the individual alkaloids $(17,18,20,23,28)$.

Real sample analysis. The lycopsamine-type alkaloids echimidine, lycopsamine, and intermedine were the most often detected, while senecionine-type PAs had slightly lower incidence rates, which is consistent with results reported for EU honey by Huybrechts and Callebaut (19). Also, Dübecke et al. (8), Griffin et al. (17), and Martinello et al. $(24,25)$ reported that among detected PAs in EU honey, the most abundant alkaloids were echimidine and lycopsamine. The obtained results are also in line with an EFSA report published in 2016. In the report it was concluded that echimidine and lycopsamine were the most important PAs in terms of contribution to the levels of PAs in honey, followed by senecionine and intermedine.

Most of the analysed monofloral honeys were free of PAs, and the highest determined concentration of $52.4 \mu \mathrm{g} \mathrm{kg}^{-1}$ was found in polyfloral honey sample (Fig. 3). The concentrations of detected alkaloids in Polish honey samples are also consistent with the results of other authors reporting PA contamination in honey of European origin. The content of PAs in honey reported by the authors was in a range of $0.6-43 \mu \mathrm{g} \mathrm{kg}^{-1}$, excluding significantly higher concentrations in honey from Italy and Spain $(3,8,18,22,25,28)$. The results also concur with previously conducted studies concerning the occurrence of PAs in Polish honey. The mean concentration and median for all samples were 2.9 and $1.6 \mu \mathrm{g} \mathrm{kg} \mathrm{kg}^{-1}$, respectively (21). However, the percentage of positive samples was higher at $68 \%$ compared to the $32 \%$ for this study. This could be caused by the different rates of contamination with PAs that often depend on the particular year of honey production or by the fact that GC-MS sum parameter method detects almost all 1,2-unsaturated PAs, while in the case of LC-MS method some PAs can remain undetected.

For the assessment of the safety of the detected PAs concentration in honey for the consumers, usually an average adult weight of $60 \mathrm{~kg}$ and an average child weight of $15 \mathrm{~kg}$, an average consumption of $20 \mathrm{~g}$, and a recommended maximum intake of PAs in the amount of $0.007 \mu \mathrm{g} \mathrm{kg}^{-1}$ of body weight (b.w.) per day are taken into consideration. The maximum intake of PAs has been proposed by EFSA, the Committee on Toxicity and the Federal Institute of Risk Assessment. The level was 
calculated according to a $\mathrm{BMDL}_{10}$ (the lower confidence limit on the benchmark dose associated with $10 \%$ response) of $73 \mu \mathrm{g} \mathrm{kg}^{-1}$ b.w. per day that was the result of a carcinogenicity study of lasiocarpine in rats, and with the MOE (margin of exposure) of 10,000.

Considering the above, adults can have a maximum daily intake of PAs of $0.42 \mu \mathrm{g}$, and children's maximum PAs daily intake should not exceed $0.105 \mu \mathrm{g}$. Thus, the maximum concentration in honey should not exceed $21 \mu \mathrm{g} \mathrm{kg}^{-1}$ for adults and $5.25 \mu \mathrm{g} \mathrm{kg}^{-1}$ for children. However, in the case of children the average consumption of $20 \mathrm{~g}$ may be somewhat overestimated. That is why data provided in the EFSA report (14) were taken for the calculation of allowable content of PAs in honey. In the young honey-consuming population, the average habitual consumption of honey has been evaluated as $0.98 \mathrm{~g} \mathrm{~kg}^{-1}$ b.w. per day. In that case, the maximum content of PAs in honey should not exceed $7.1 \mu \mathrm{g} \mathrm{kg}^{-1}$.

Amongst the analysed honey samples, only one would exceed the limit of recommended daily intake if consumed in the amount of $20 \mathrm{~g}$ or higher, in an adult diet. Other positive samples had relatively low PAs concentrations ranging from 1.4 to $9.7 \mu \mathrm{g} \mathrm{kg}^{-1}$ and can be regarded as safe under the $20 \mathrm{~g}$ consumption amount. In a child diet, two samples would exceed the limit of PAs content of $7.1 \mu \mathrm{g} \mathrm{kg}^{-1}$

However, in an EFSA report from 2017, the CONTAM Panel selected the BMDL 10 of $237 \mu \mathrm{g} \mathrm{kg}^{-1}$ b.w. per day, determined on the basis of incidence of liver haemangiosarcoma in female rats exposed to riddelliine (15). Using the new $\mathrm{BMDL}_{10}$ the maximum intake can be calculated as three times higher than the previous one. Consequently, the concentration of PAs in honey can also be three times higher, which would be $71.1 \mu \mathrm{g} \mathrm{kg}^{-1}$ and $24.2 \mu \mathrm{g} \mathrm{kg}^{-1}$ for adults and children, respectively, and still be treated as safe. With these limits, no PA-positive honeys would exceed the recommended limit of daily intake by adults in their $20 \mathrm{~g}$ per day consumption, and only one honey would pose a potential risk to children.

In conclusion, a sensitive and selective method suitable for determination of PAs in honey has been developed. The method has been validated according to the SANTE/11945/2015 guidance document, and all evaluated parameters are in agreement with the requirements. For these reasons the developed method can be perceived as a useful tool for the analysis of selected PAs in honey in routine laboratory practice. The method has been applied to the analysis of 50 honey samples. Among analysed samples $32 \%$ were positive for the presence of at least one of the alkaloids. Echimidine, lycopsamine, and intermedine were the most abundant among detected alkaloids. On the basis of the detected PAs concentrations it can be stated that most of the analysed honey samples should not pose any potential risk to the consumers.

Conflict of Interests Statement: The authors declare that there is no conflict of interests regarding the publication of this article.
Financial Disclosure Statement: The study was supported by the National Veterinary Research Institute, Pulawy, Poland.

Animal Rights Statement: None required.

Acknowledgements: The authors are grateful to Tomasz Grenda, Tomasz Kiljanek, Tomasz Błądek, and Agnieszka Nawrocka for sharing the honey samples used in the study.

\section{References}

1. Beales K.A., Betteridge K., Colegate S.M., Edgar J.A.: Solidphase extraction and LC-MS analysis of pyrrolizidine alkaloids in honeys. J Agric Food Chem 2004, 52, 6664-6672.

2. Betteridge K., Cao Y., Colegate S.M.: Improved method for extraction and LC-MS analysis of pyrrolizidine alkaloids and their N-oxides in honey: application to Echium vulgare honeys. J Agric Food Chem 2005, 53, 1894-1902.

3. Bodi D., Ronczka S., Gottschalk C., Behr N., Skibba A., Wagner M., Lahrssen-Wiederholt M., Preiss-Weigert A., These A.: Determination of pyrrolizidine alkaloids in tea, herbal drugs, and honey. Food Addit Contam Part A 2014, 31, 1886-1895.

4. Boppré M.: The ecological context of pyrrolizidine alkaloids in food, feed, and forage: an overview. Food Addit Contam Part A 2011, 28, 260-281

5. Boppré M., Colegate S.M., Edgar J.A.: Pyrrolizidine alkaloids of Echium vulgare honey found in pure pollen. J Agric Food Chem 2005, 53, 594-600.

6. Chung S.W.C., Lam A.C.H.: Investigation of pyrrolizidine alkaloids including their respective $\mathrm{N}$-oxides in selected food products available in Hong Kong by liquid chromatography electrospray ionisation mass spectrometry. Food Add Contam Part A 2017, 34, 1184-1192.

7. Cramer L., Beuerle T.: Detection and quantification of pyrrolizidine alkaloids in antibacterial medical honeys. Planta Med 2012, 78, 1976-1982.

8. Dübecke A., Beckh G., Lüllmann C.: Pyrrolizidine alkaloids in honey and bee pollen. Food Addit Contam Part A 2011, 28, 348-358.

9. Edgar J.A., Colegate S.M., Boppre M., Molyneux R.J.: Pyrrolizidine alkaloids in food: a spectrum of potential health consequences. Food Addit Contam Part A 2011, 28, 308-324.

10. Edgar J.A., Molyneux R.J., Colegate S.M.: Pyrrolizidine alkaloids: potential role in the etiology of cancers, pulmonary hypertension, congenital anomalies, and liver disease. Chem Res Toxicol 2015, 28, 4-20.

11. Edgar J.A., Roeder E., Molyneaux R.J.: Honey from plants containing pyrrolizidine alkaloids: a potential threat to health. J Agric Food Chem 2002, 50, 2719-2730.

12. El Hawari K., Mokh S., Doumyati S., Al Iskandarani M., Verdon E.: Development and validation of a multiclass method for the determination of antibiotic residues in honey using liquid chromatography-tandem mass spectrometry. Food Add Contam Part A 2017, 34, 582-597.

13. European Food Safety Authority, EFSA Panel on Contaminants in the Food Chain (CONTAM). Scientific opinion on pyrrolizidine alkaloids in food and feed. EFSA J 2011, 9, 2406, 1-134.

14. European Food Safety Authority (EFSA). Dietary exposure assessment to pyrrolizidine alkaloids in the European population. EFSA J 2016, 14, 4572, 1-50.

15. European Food Safety Authority, EFSA Panel on Contaminants in the Food Chain (CONTAM). Risks for human health related to the presence of pyrrolizidine alkaloids in honey, tea, herbal infusions and food supplements. EFSA J 2017, 15, 4908, 1-34.

16. European Food Safety Authority. Summary report of the standing committee on the food chain and animal health, held in Brussels 
on 11 July 2012 , section toxicological safety of the food chain. 2012, http://www.efsa.europa.eu/en/efsajournal/doc/2406.pdf.

17. Griffin C.T., Danaher M., Elliott C.T., Kennedy D.G., Furey A.: Detection of pyrrolizidine alkaloids in commercial honey using liquid chromatography-ion trap mass spectrometry. Food Chem 2013, 136, 1577-1583.

18. Griffin C.T., O’Mahony J., Danaher M., Furey A.: Liquid chromatography tandem mass spectrometry detection of targeted pyrrolizidine alkaloids in honeys purchased within Ireland. Food Anal Methods 2015, 8, 18-31.

19. Huybrechts B., Callebaut A.: Pyrrolizidine alkaloids in food and feed on the Belgian market. Food Addit Contam Part A 2015, 32, 1939-1951.

20. Kempf M., Beuerle T., Bühringer M., Denner M., Trost D., von der Ohe K., Bhavanam V.B.R., Schreier P.: Pyrrolizidine alkaloids in honey: Risk analysis by gas chromatography-mass spectrometry. Mol Nutr Food Res 2008, 52, 1193-1200.

21. Kowalczyk E., Sieradzki Z., Kwiatek K.: Determination of pyrrolizidine alkaloids in honey with sensitive gas chromatography-mass spectrometry method. Food Anal Methods 2018, 11, 1345-1355.

22. Lucatello L., Merlanti R., Rossi A., Montesissa C., Capolongo F.: Evaluation of some pyrrolizidine alkaloids in honey samples from the Veneto Region (Italy) by LC-MS/MS. Food Anal Methods 2016, 9, 1825-1836.

23. Lucchetti M., Glauser G., Kilchenmann V., Dübecke A., Beckh G., Praz C., Kast C.: Pyrrolizidine alkaloids from Echium vulgare in honey originate primarily from floral nectar. J Agric Food Chem 2016, 64, 5267-5273.
24. Martinello M., Borin A., Stella R., Bovo D., Biancotto G., Gallina A., Mutinelli F.: Development and validation of a QuEChERS method coupled to liquid chromatography and high resolution mass spectrometry to determine pyrrolizidine and tropane alkaloids in honey. Food Chem 2017, 234, 295-302.

25. Martinello M., Cristofoli C., Gallina A., Mutinelli F.: Easy and rapid method for the quantitative determination of pyrrolizidine alkaloids in honey by ultra-performance liquid chromatographymass spectrometry: An evaluation in commercial honey. Food Control 2014, 37, 146-152.

26. Mulder P.P.J., López Sánchez P., These A., Preiss-Weigert A., Castellari M.: Occurrence of pyrrolizidine alkaloids in food 2015, EFSA:EN-859.

27. Mulder P.P.J., de Witte S.L., Stoopen G.M., van der Meulen J., van Wikselaar P.G., Gruys E., Groot M.J., Hoogenboom R.L. Transfer of pyrrolizidine alkaloids from various herbs to eggs and meat in laying hens. Food Addit Contam Part A 2016, 33, 1826-1839.

28. Orantes-Bermejo F.J., Serra Bonvehíb J., Gómez-Pajueloc A., Megíasa M., Torresa C.: Pyrrolizidine alkaloids: their occurrence in Spanish honey collected from purple viper's bugloss (Echiumspp.). Food Addit Contam Part A 2013, 30, 1799-1806.

29. SANTE/11945/2015 Guidance document on analytical quality control and method validation procedures for pesticides residues analysis in food and feed. Supersedes SANCO/12571/2013, Implemented by 01/01/2016, 1-42.

30. Statistical manual of AOAC - Association of official analytical chemistry, Edited by Youden W.J., Steiner E.H., Washington 1975 , p. 88. 